\title{
Clinical Characteristics of Elderly Patients with COVID-19 in Hunan Province, China: A Multicenter, Retrospective Study
}

\author{
Ting Guo ${ }^{a-c}$ Qinxue Shen ${ }^{a-c}$ Wei Guo ${ }^{a-c}$ Wenlong He ${ }^{a-c}$ Jinhua Li ${ }^{a-c}$ \\ Yi Zhang $^{\mathrm{a}-\mathrm{c}}$ Yunnian Wang ${ }^{\mathrm{d}}$ Zhiguo Zhou ${ }^{\mathrm{e}}$ Dingding Deng $^{\mathrm{f}}$ Xiaoli Ouyang $^{\mathrm{a}-\mathrm{c}}$ \\ Zhi Xiang $^{g}$ Minyan Jiang $^{\text {h }}$ Minyong Liang ${ }^{i}$ Peng Huang $^{j}$ Zaimei Peng $^{k}$

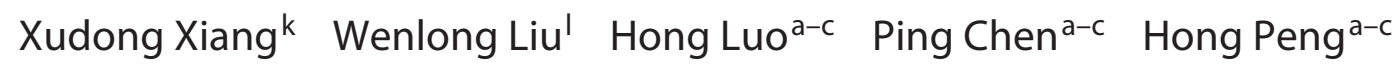 \\ a Department of Respiratory and Critical Care Medicine, The Second Xiangya Hospital of Central-South University, \\ Changsha, China; ${ }^{\mathrm{b}}$ Research Unit of Respiratory Disease, Central-South University, Changsha, China; ${ }^{\mathrm{C}}$ The Respiratory \\ Disease Diagnosis and Treatment Center of Hunan Province, Changsha, China; ${ }^{d}$ Department of Respiratory and \\ Critical Care Medicine, The Traditional Chinese Medicine Hospital of Liling, Zhuzhou, China; ' Department of \\ Respiratory and Critical Care Medicine, The First Hospital of Changsha, Changsha, China; ${ }^{f}$ Department of Respiratory \\ and Critical Care Medicine, The First Hospital of Shaoyang University, Shaoyang, China; ${ }^{9}$ Department of Respiratory \\ and Critical Care Medicine, The First People's Hospital of Huaihua, Huaihua, China; ${ }^{\mathrm{h}}$ Department of Respiratory and \\ Critical Care Medicine, The Central Hospital of Xiangtan, Xiangtan, China; 'Department of Respiratory and Critical Care \\ Medicine, The People's Hospital of Xiangxi Autonomous Prefecture, Xiangxi, China; 'Department of Respiratory and \\ Critical Care Medicine, The Central Hospital of Zhuzhou, Zhuzhou, China; ${ }^{k}$ Department of Emergency, The Second \\ Xiangya Hospital of Central-South University, Changsha, China; 'Department of Respiratory and Critical Care Medicine, \\ The Junshan People's Hospital of Yueyang, Yueyang, China
}

\section{Keywords}

Elderly · Epidemiology · Older people · Coronavirus disease 2019. SARS-CoV-2

\begin{abstract}
Background: The aim of this study was to analyze and summarize the clinical characteristics of elderly patients with coronavirus disease 2019 (COVID-19) and compare the differences of young-old patients (60-74 years old) and old-old patients ( $\geq 75$ years old). Methods: In this retrospective, multicenter study, the medical records of elderly patients who were diagnosed with COVID-19 in Hunan province, China, from January 21 to February 19, 2020 were reviewed. The characteristics of young-old patients and old-old patients
\end{abstract}

were compared. Results: Of the 105 elderly patients confirmed with COVID-19, 81.0\% were young-old patients, and $19.0 \%$ were old-old patients; $54.3 \%$ of elderly patients were females. Overall, $69.5 \%$ of elderly patients had underlying diseases, and the most common comorbidities included hypertension $(43.8 \%)$, diabetes $(25.7 \%)$, and cardiac disease (16.2\%). Of the elderly patients, $22.9 \%$ were severe and $10.5 \%$ were critical severe cases. On admission, the most frequent symptoms in elderly patients included fever (66.7\%), cough (64.8\%), and fatigue (33.3\%). Lymphopenia (31.4\%), increased D-dimer (38.1\%), depressed albumin (36.2\%), elevated lactate dehydrogenase (41.0\%), and a high level of Creactive protein $(79.0 \%)$ were common among elderly patients with COVID-19. The median prothrombin time (PT) and the activated partial thromboplastin time (APTT) were karger@karger.com

www.karger.com/ger

Karger (c) 2020 S. Karger AG, Base

(1)

\footnotetext{
Hong Peng or Ping Chen

Department of Respiratory and Critical Care Medicine

The Second Xiangya Hospital of Central-South University

No. 139 Renmin Middle Road, Changsha, Hunan 410011 (China)

penghong66@csu.edu.cn or pingchen0731@csu.edu.cn
} 
longer in old-old patients than young-old patients (PT median 12.3 vs. $13.1 \mathrm{~s}, p=0.007$; APTT median 39.0 vs. $33.5 \mathrm{~s}$, $p=0.045$ ). Young-old patients showed fewer complications (14.1\%) than old-old patients $(40.0 \% ; p=0.0014)$ and fewer received invasive ventilator support ( $3.5 \mathrm{vs.} 25.0 \%, p=0.006$ ). As of March 11, 2020, 85.7\% of elderly patients had been discharged, 3 deaths had occurred, and $11.4 \%$ were still hospitalized. Conclusions: Elderly patients usually have chronic medical illness and are likely to have a severe or critically severe condition. They could show atypical symptoms without fever or cough and multiple organ dysfunction. Old-old patients tend to have more complications than young-old patients during hospitalization. Careful nursing, observation, and systemic treatment are very important in elderly patients.

c) 2020 S. Karger AG, Basel

\section{Introduction}

Severe acute respiratory syndrome coronavirus 2 (SARS-CoV-2) is a novel type of beta coronavirus with high pathogenicity to the human being, and has resulted in the large-scale transmission of novel coronavirus disease 2019 (COVID-19) worldwide since December 2019 from Wuhan, China [1]. The most common clinical manifestations of COVID-19 include fever, cough, dyspnea, fatigue, and myalgia. A few patients have developed severe pneumonia and they may present with acute respiratory distress syndrome (ARDS), extrapulmonary organ dysfunction, or even death [2]. Thus far, over 700,000 people have been infected with SARS-CoV-2 and more than 30,000 patients have died of COVID-19 globally [3]. The World Health Organization (WHO) declared the COVID-19 outbreak a global pandemic on March 12, 2020 [4].

According to an early clinical report including 138 COVID-19 cases in Wuhan [5], patients admitted to the intensive care unit (ICU) were older than non-ICU patients. In a study enrolling 52 critically ill Wuhan patients [6], the average age of non-survivors was 64.6 years. Another recent study from Wuhan also indicates that old age is a risk factor for in-hospital death [7]. Outside of Wuhan, research from Beijing showed there were more severe cases among patients aged over 65 years [8]. All these studies suggest the elderly are more susceptible to COVID-19 and likely to have poor outcomes. However, at present, information regarding the clinical characteristics of elderly COVID-19 patients is limited, and the features and differences in young-old (60-74 years old) and old-old ( $\geq 75$ years old) patients are unknown. In this multicenter study, we aim to summarize the clinical features and outcomes of elderly COVID-19 patients in Hunan province, a neighbor of Hubei province, and compare the difference between young-old patients and old-old patients.

\section{Materials and Methods}

\section{Study Design and Participants}

In this multicenter, retrospective study, patients laboratory confirmed as having SARS-CoV-2 infection according to WHO interim guidance [9] and aged 60 years and older from eight different regions, including Changsha City, Zhuzhou City, Huaihua City, Shaoyang City, Xiangtan City, Yueyang City, Zhangjiajie City, and Xiangxi Autonomous Prefecture in Hunan province, China, were enrolled. The designated hospitals in these cities for COVID-19 treatment included the Public Health Clinic Center of Changsha City, the Lvkou People's Hospital of Zhuzhou City, the First People's Hospital of Huaihua City, the Central Hospital of Shaoyang City, the Central Hospital of Xiangtan City, the Junshan People's Hospital of Yueyang City, the People's Hospital of Zhangjiajie City, the Longshan People's Hospital in Xiangxi Autonomous Prefecture, and the Yongshun People's Hospital in Xiangxi Autonomous Prefecture. The first case with COVID-19 was confirmed on January 21, 2020, in Hunan province. The medical records of elderly COVID-19 patients who were referred to these hospitals from January 21 to February 19, 2020 were retrospectively reviewed and analyzed. Clinical outcomes were followed up to March 11,2020. The elderly patients were divided into two age groups, including the young-old COVID-19 group (60-74 years old) and the old-old COVID-19 group ( $\geq 75$ years old).

Epidemiological information, signs and symptoms on admission, laboratory as well as radiological results, and treatment and prognosis data were obtained from medical records. Any missing or uncertain records were collected and clarified through direct communications with patients or their families. All data were double checked by 2 independent physicians (J.L. and W.G.). Clinical features and outcomes of the two age groups were compared.

\section{Definitions}

Based on the New Coronavirus Pneumonia Prevention and Control Program (version seven) [10] from the National Health Commission of China, patients with SARS-CoV-2 infection were divided into asymptomatic carriers, mild patients, moderate patients, severe patients, and critically severe patients (Table 1). According to the guideline, asymptomatic carriers were not classified as confirmed cases. In our study, asymptomatic carriers were not included in any group. The patients with a history of exposure to Wuhan within 14 days before illness onset were identified as tourists or residents of Wuhan.

\section{Statistical Analysis}

Statistical analyses were performed with SPSS (version 26.0). Continuous variables are described as the median (interquartile range, IQR). Categorical variables are presented as $n(\%)$. The median (IQR) was compared with the Mann-Whitney U test. Categorical variables were compared by the $\chi^{2}$ test or Fisher's exact test. $p<0.05$ was considered statistically significant. 
Table 1. Different clinical types of patients with SARS-CoV-2 infection

\begin{tabular}{|c|c|}
\hline Types & Characteristics \\
\hline Asymptomatic carriers ${ }^{1}$ & $\begin{array}{l}\text { Laboratory-confirmed SARS-CoV-2 infection without symptoms and imaging } \\
\text { findings }\end{array}$ \\
\hline Mild & Mild clinical symptoms without imaging findings of pneumonia \\
\hline Moderate & Fever or respiratory symptoms with imaging findings of pneumonia \\
\hline Severe & $\begin{array}{l}\text { Meet any of the following: } \\
\text { 1. Respiratory distress with respiratory frequency } \geq 30 \text { breaths } / \mathrm{min} \\
\text { 2. Pulse oximeter oxygen saturation }\left(\mathrm{SpO}_{2}\right) \leq 93 \% \text { in resting state } \\
\text { 3. } \mathrm{PaO}_{2} / \mathrm{FiO}_{2} \leq 300 \mathrm{~mm} \mathrm{Hg}(1 \mathrm{~mm} \mathrm{Hg}=0.133 \mathrm{kPa}) \\
\text { 4. Showing a rapid progression }(>50 \%) \text { on } \mathrm{CT} \text { imaging within } 24-48 \mathrm{~h}\end{array}$ \\
\hline Critical severe & $\begin{array}{l}\text { Meet any of the following: } \\
\text { 1. Respiratory failure in need of mechanical ventilation } \\
\text { 2. Shock } \\
\text { 3. With other organ dysfunction }\end{array}$ \\
\hline
\end{tabular}

${ }^{1}$ Asymptomatic carriers were not classified as confirmed cases of COVID-19.

\section{Results}

\section{Epidemiological Features}

By February 19, 2020, a total of 579 admitted hospital patients were identified as laboratory-confirmed SARSCoV-2 infection cases in these nine hospitals in Hunan province. Fourteen patients were asymptomatic carriers. There were 107 patients aged 60 years and over. Only 2 elderly cases were asymptomatic carriers. Among confirmed cases with COVID-19, the proportion of confirmed elderly patients was $18.6 \%$ (105/565). Baseline data for all 105 confirmed patients aged above 60 years are shown in Table 2. The median age of all elderly patients was 67.0 years (IQR 64.0-74.0); 57 (54.3\%) elderly patients were female in our cohort. Overall, $69.5 \%$ of elderly patients had chronic medical illness, and the most common comorbidities included hypertension (43.8\%), diabetes $(25.7 \%)$, and cardiac disease (16.2\%). On admission, $66.7 \%$ of elderly patients showed fever, $64.8 \%$ had cough, and $33.3 \%$ had fatigue. Other symptoms included dyspnea $(29.5 \%)$, diarrhea $(9.5 \%)$, anorexia $(8.6 \%)$, headache $(8.6 \%)$, myalgia $(7.6 \%)$, and vomiting $(5.7 \%)$. The median time of incubation in elderly patients was 8.0 days (IQR 4.0-15.0). The median time from the onset of symptoms to hospital admission was 5.0 days (IQR 2.0-8.0). Regarding clinical types according to the severity of disease, $61.9 \%$ of elderly patients were moderate, $22.9 \%$ were severe, and 10.5\% were critical. Among 105 elderly patients, $85(81.0 \%)$ were young-old patients and $20(19.0 \%)$ were old-old cases. There were no differences regarding sex, smoking history, comorbidities, family cluster cases, clinical types, signs and symptoms on admission, and time of incubation or days from onset of symptoms to admission between the young-old group and old-old group.

\section{Clinical Characteristics and Outcomes}

On admission, the median leukocyte levels were $4.9 \times$ $10^{9} / \mathrm{L}$ (IQR 3.8-6.5) in elderly patients and leukocytes below the normal range were in $30.5 \%$ in elderly cases (Table 3). In elderly patients, lymphocytes were below the normal range in $31(31.4 \%)$ and $38.1 \%$ had an elevated D-dimer level. Lactate dehydrogenase ( $\mathrm{LDH}$ ) was above the normal range in 43 (41.0\%). Of the elderly patients, $79.0 \%$ showed increased C-reactive protein (CRP) levels, and $88.7 \%$ had images of bilateral pneumonia on admission. The median prothrombin time (PT) was longer in old-old patients compared with young-old patients (median 12.3 s [IQR 11.2-12.9] vs. 13.1 s [IQR, 12.4-13.4]; $p=0.007)$. The activated partial thromboplastin time (APTT) in old-old patients was longer than in young-old patients (median $39.0 \mathrm{~s}$ [IQR 29.9-43.5] vs. $33.5 \mathrm{~s}$ [IQR, 30.3-37.3]; $p=0.045$ ). Levels of LDH were increased in $14(47.1 \%)$ young-old patients, and this proportion was lower in the old-old group (15.0\%; $p=0.011)$. Old-old patients showed a higher median level of creatine than young-old patients (median 78.7 U/L [IQR 66.9-106.5] vs. $64.5 \mathrm{U} / \mathrm{L}$ [IQR 52.4-77.8]; $p=0.003$ ). More old-old 
Table 2. Demographics and baseline characteristics of elderly patients with SARS-CoV-2 infection

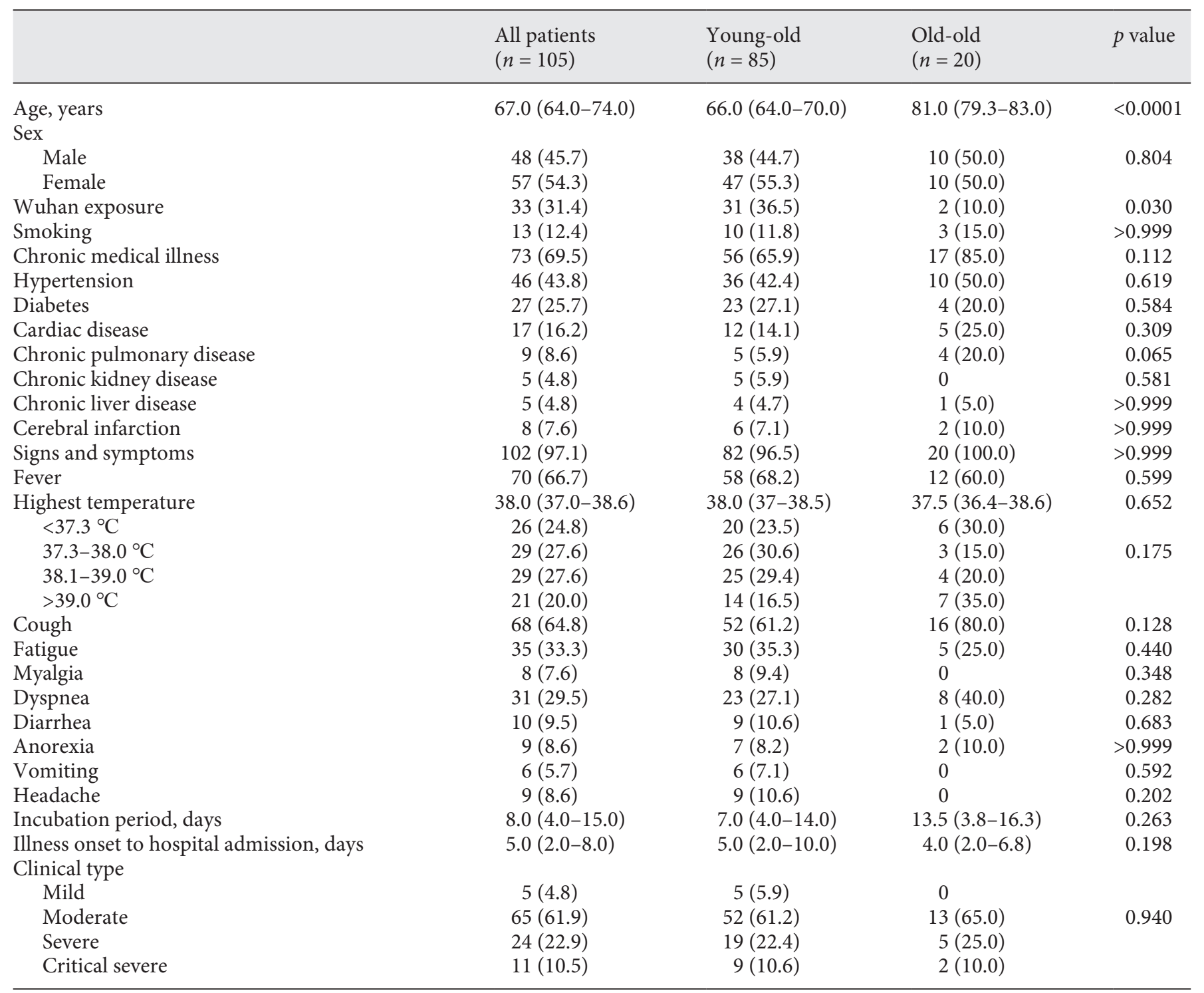

Data are presented as the median (IQR) or $n(\%)$.

patients $(25.0 \%)$ had elevated creatine than young-old patients $(7.1 \% ; p=0.033)$. Old-old patients showed a higher median level of blood urea nitrogen than youngold patients (median $7.7 \mathrm{mmol} / \mathrm{L}$ [IQR 4.9-8.6] vs. 5.2 $\mathrm{mmol} / \mathrm{L}$ [IQR 4.1-6.7]; $p=0.005$ ).

During the hospitalization, $19.0 \%$ of elderly patients had complications, including ARDS (10.5\%), acute cardiac injury (4.8\%), acute kidney injury (4.8\%), acute hepatic injury (1.0\%), sepsis $(5.7 \%)$, allergic eruption (1.9\%), and pneumothorax (1.0\%; Table 4). Overall, $93.3 \%$ of elderly patients received antiviral therapy, $60 \%$ used antibiotics, $48.6 \%$ used corticosteroid, and $42.9 \%$ received intravenous immunoglobulin. Young-old patients showed fewer complications (14.1\%) compared to old-old patients $(40.0 \% ; p=0.0014)$. Old-old patients required invasive mechanical ventilator support more than young-old patients ( 25.0 vs. $3.5 \%, p=0.045)$. As of March 11, 2020, 85.7\% of elderly patients had been discharged and 3 deaths had occurred. There were no differences in the discharge rates and mortality between the young-old and old-old groups. The time of negative RT-PCR results and days from admission to discharge were not statistically significance between the two groups. 
Table 3. Laboratory and image findings of elderly patients infected with SARS-CoV-2 on admission to hospital

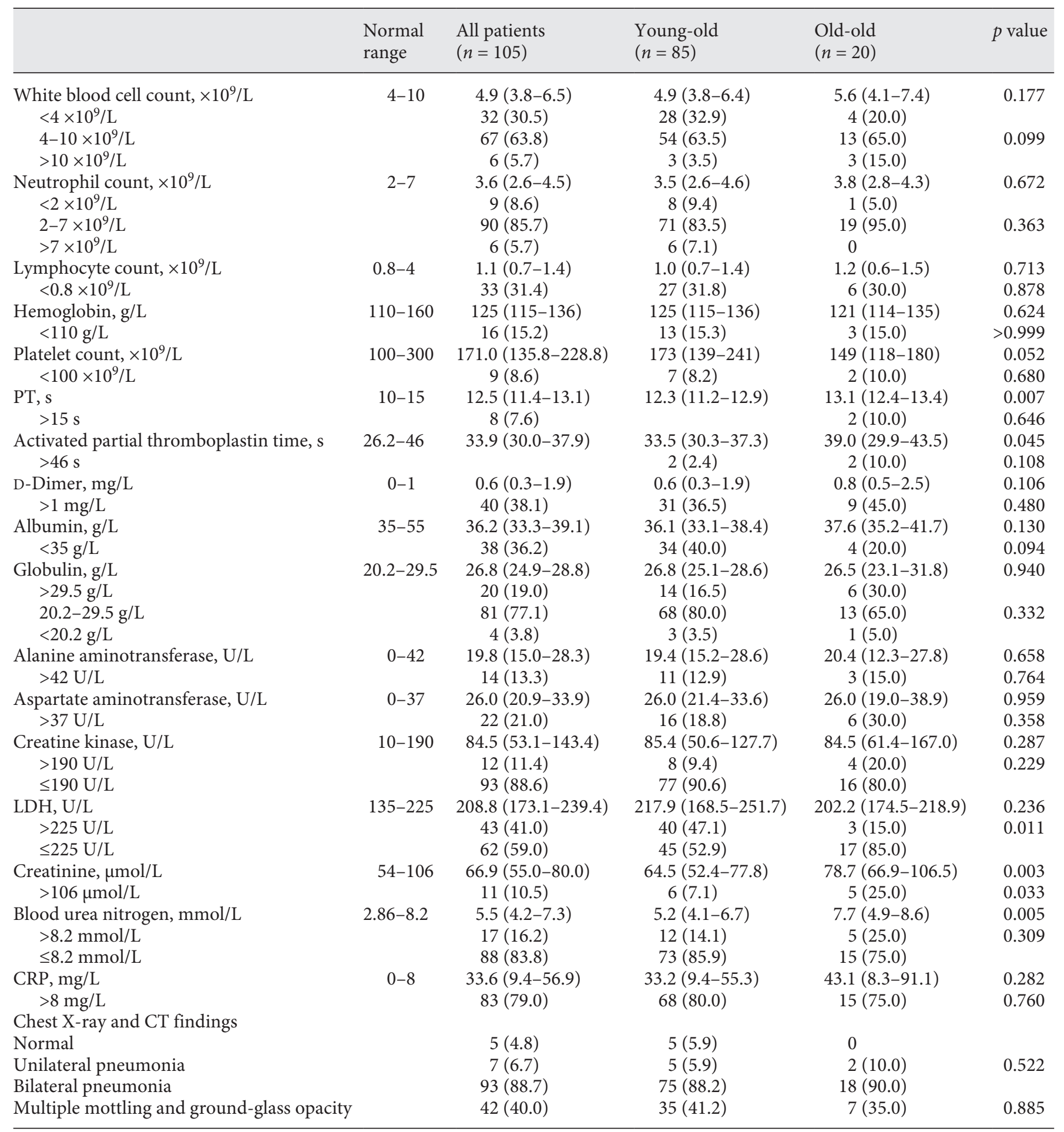

Data are presented as the median (IQR) or $n(\%)$. PT, prothrombin time; LDH, lactate dehydrogenase; CRP, C-reactive protein. 
Table 4. Treatment and outcomes of elderly patients infected with SARS-CoV-2

\begin{tabular}{|c|c|c|c|c|}
\hline & $\begin{array}{l}\text { All patients } \\
(n=105)\end{array}$ & $\begin{array}{l}\text { Young-old } \\
(n=85)\end{array}$ & $\begin{array}{l}\text { Old-old } \\
(n=20)\end{array}$ & $p$ value \\
\hline Complications & $20(19.0)$ & $12(14.1)$ & $8(40.0)$ & 0.0014 \\
\hline ARDS & $11(10.5)$ & $5(5.9)$ & $6(30.0)$ & 0.0003 \\
\hline Acute cardiac injury & $5(4.8)$ & $1(1.2)$ & $4(20.0)$ & 0.002 \\
\hline Acute kidney injury & $5(4.8)$ & $2(2.4)$ & $3(15.0)$ & 0.028 \\
\hline Acute hepatic injury & $1(1.0)$ & $1(1.2)$ & 0 & $>0.9999$ \\
\hline Sepsis & $6(5.7)$ & $2(2.4)$ & $4(20.0)$ & 0.006 \\
\hline Allergic eruption & $2(1.9)$ & $2(2.4)$ & 0 & $>0.9999$ \\
\hline Pneumothorax & $1(1.0)$ & 0 & $1(5.0)$ & 0.160 \\
\hline \multicolumn{5}{|l|}{ Treatment } \\
\hline Antiviral therapy & $98(93.3)$ & $79(92.9)$ & $19(95.0)$ & $>0.999$ \\
\hline Antibiotic therapy & $63(60.0)$ & $53(62.4)$ & $10(50.0)$ & 0.447 \\
\hline Corticosteroid & $51(48.6)$ & $42(49.4)$ & $9(45.0)$ & 0.806 \\
\hline Intravenous immunoglobulin therapy & $45(42.9)$ & $37(43.5)$ & $8(40.0)$ & 0.808 \\
\hline Oxygen support & $89(84.8)$ & $71(83.5)$ & $18(90.0)$ & 0.527 \\
\hline Nasal cannula & $67(63.8)$ & $57(67.1)$ & $10(50.0)$ & 0.197 \\
\hline High-flow nasal cannula & $8(7.6)$ & $7(8.2)$ & $1(5.0)$ & 0.702 \\
\hline Non-invasive ventilation & $7(6.7)$ & $5(5.9)$ & $2(10.0)$ & 0.616 \\
\hline Invasive mechanical ventilation & $8(7.6)$ & $3(3.5)$ & $5(25.0)$ & 0.006 \\
\hline ECMO & $3(2.9)$ & $1(1.2)$ & $2(10.0)$ & 0.092 \\
\hline \multicolumn{5}{|l|}{ Prognosis } \\
\hline Discharge & $90(85.7)$ & $75(88.2)$ & $15(75.0)$ & 0.744 \\
\hline Death & $3(2.9)$ & $1(1.2)$ & $2(10.0)$ & 0.066 \\
\hline Onset of symptoms to negative RT-PCR results, days & $18.0(14.0-24.8)$ & $18.0(14.0-24.0)$ & $18.0(12.0-27.0)$ & 0.870 \\
\hline Onset of admission to discharge, days & $17.0(13.0-23.0)$ & $17.0(13.0-20.0)$ & $20.0(14.0-27.0)$ & 0.359 \\
\hline
\end{tabular}

Data are presented as the median (IQR) or $n(\%)$. ARDS, acute respiratory distress syndrome.

\section{Discussion}

A total of 105 elderly patients aged above 60 years and confirmed as having SARS-CoV-2 infection were included in the present study, and these patients came from eight different cities in Hunan province, China. The proportion of these elderly patients was $18.6 \%$ in all cases confirmed with SARS-CoV-2 infection in this multicenter study in Hunan province. The proportion of elderly patients ranged from 11.25 to $37 \%$ in different studies, including within Wuhan those outside of Wuhan $[8$, $11,12]$. Notably, the proportion of elderly patients was higher in Wuhan compared to other regions, and our study further confirmed this phenomenon, which indicates elderly people are vulnerable to COVID-19, and with increased caution and adoption of home quarantine the infection rate in elderly people could be reduced. Although some studies have presented the proportion of elderly patients with COVID-19, the clinical and epidemiologic information in this age group is very limited. Our study aimed to provide a comprehensive introduc- tion of epidemiological and clinical characteristics of elderly patients with COVID-19 outside of Wuhan. Moreover, our study further compared the difference between young-old patients (60-74 years old) and old-old patients ( $\geq 75$ years old). Among all the elderly patients, $81.0 \%$ were young-old patients and $19.0 \%$ were old-old patients in our cohort. As for demographic information, fewer old-old patients had a Wuhan exposure history, which may be explained by a reduction of traveling with greater age.

In the present study, more than half of the elderly patients were female. From early studies in Wuhan, 54.3$68 \%$ of COVID-19 patients were male $[5,12]$. Outside of Wuhan, some studies showed an approximate 1:1 ratio of females to males, such as $51.5 \%$ of female patients in Beijing [8] and $51.3 \%$ in Jiangsu [11]. In our cohort, there were more female patients, which may be due to the limited sample size. A large investigation is needed to explore whether there is a gender difference in COVID-19, especially in the elderly group. Many elderly patients in our cohort had chronic medical illness, and the most frequent 
comorbidities were hypertension, diabetes, and cardiac disease. The elderly patients with comorbid conditions were more likely to progress to severe illness [6], so the management of chronic disease is vital in elderly patients with COVID-19, with special attention, in our experience, on monitoring and controlling blood pressure and glucose. Fever, cough, and fatigue were the most common symptoms in elderly patients on admission. Although fever and cough were the most frequent symptoms in elderly patients on admission in our study, the ratios were relatively lower than in other studies including patients of all ages, which were 77-96 and 75-81\%, respectively [1315]. This suggests some elderly patients may not present with typical symptoms, such as fever or cough, like young patients when infected with SARS-CoV-2. A previous report indicated fever might be blunted or even absent in elderly patients with bacterial or viral infection, because of a low basal temperature, disturbance in thermal homeostasis by aging, and frequent use of medications, such as aspirin [16]. Elderly patients with COVID-19 may only have fatigue, myalgia, headache, or digestive symptoms, including anorexia, vomiting without fever, or cough. Therefore, clinical care for elderly patients should be aware of these non-classical presentations. Moreover, the incidence of severe or critically severe illness was high in elderly patients, irrespective of the young-old or old-old patient categories, as nearly one third elderly patients were severe or critically severe cases. Combined with chronic medical illnesses, the possibility of atypical symptoms, and high incidence of severe illness, elderly patients require much more attention and nursing during the $\mathrm{CO}$ VID-19 pandemic.

In terms of laboratory results, more than half of elderly patients had normal white blood cells, and nearly one third of elderly patients had reduced white blood cells and lymphocytes. Lymphopenia was a common abnormality in patients with SARS-CoV-2 infection, occurring in 32.5-75.4\% of COVID-19 patients from different studies [11-14]. Depressed lymphocytes were also a common finding in two other beta coronavirus infections, namely severe acute respiratory syndrome coronavirus (SARS$\mathrm{CoV}$ ) and Middle East respiratory syndrome coronavirus (MERS-CoV) [17, 18]. From a large study including 1,068 healthy adults, it was shown that the circulating naïve CD4+ and CD8+ T cells would decrease with aging, which is a sign of immunosenescence of the immune system [19]. A recent study also indicated a lower level of CD4+ and CD8+ T cells in severe COVID-19 patients compared with non-severe cases [20]. The results of the two studies may explain the phenomenon of a higher incidence of

Features of Elderly Patients with COVID-19 severe COVID-19 in elderly patients, partly due to T cellular immune deficiency.

Other laboratory abnormalities, such as increased D-dimer, depressed albumin, elevated LDH, and a high level of CRP were common among elderly patients with COVID-19. D-Dimer is a specific marker of fibrinolysis and has been used to screen patients with venous thromboembolism, but it also increases in inflammation and could predict a poor outcome in sepsis $[21,22]$. In total, $38.1 \%$ of elderly patients had an increased D-dimer level in our cohort, but no subjects were diagnosed with venous thromboembolism during the hospitalization, which suggests the elevated D-dimer was more likely to be associated with inflammation in the infection of SARSCoV-2. The abnormal albumin and LDH suggest that SARS-CoV-2 infection is associated with extrapulmonary organ injury, including hepatic injury or myocardia injury. A recent study indicated a high proinflammatory cytokine storm (including high levels of IL-6, IL-10, IL-2, and IFN- $\gamma$ ) in severe COVID-19 patients [23]. As many hospitals in our study could not test these cytokines, we did not record the levels of cytokines in elderly patients and only used CRP to reflect inflammation. CRP has an important role in innate immunity against infections [24]. The median level of CRP in elderly patients from our study was $33.6 \mathrm{mg} / \mathrm{L}$ and $79.0 \%$ of elderly patients had an increased CRP, which indicated the intensity of the inflammatory process during SASR-CoV-2 infection. Apparently, the infection of SAR-CoV-2 could induce systemic damage to a patient's body. It is important to monitor organ function and the inflammatory response with laboratory tests, especially in elderly patients with COVID-19.

Comparing the laboratory results between young-old and old-old patients, old-old patients had a longer PT as well APTT, and higher levels of creatine and blood urea nitrogen. In fact, some coagulation factors, such as fibrinogen, factor V, factor VII, factor VIII, factor IX, and factor XIII, increase with aging and older patients tend to have a state of hypercoagulability $[25,26]$. Based on our study, old-old patients with COVID-19 showed longer $\mathrm{PT}$ and APTT than young-old patients, suggesting that the SARS-CoV-2 infection could induce coagulation abnormalities. However, we cannot completely exclude the effect of drugs as many old patients take anticoagulants to prevent or treat coronary heart disease. Further studies are necessary to explore changes of the coagulation system in patients with COVID-19. According to our study, old-old patients with COVID-19 had higher levels of creatine and blood urea nitrogen, which could be explained 
by the loss of kidney function associated with aging [27]. It reminds us of the importance of monitoring kidney function of older patients and adopting therapy with fewer side effects on the kidney.

During hospitalization, about $40.0 \%$ of old-old patients had complications and the ratio was only $14.1 \%$ in young-old patients. More old-old patients had ARDS, acute cardiac injury, acute kidney injury, sepsis, and pneumothorax than young-old patients. Furthermore, more old-old patients received invasive mechanical ventilation compared with young-old patients. The present study indicates a higher incidence of complications in older patients, but with timely diagnosis and interventions, including effective respiratory support, old-old patients with complications could also achieve an ideal prognosis similar to young-old patients. In our cohort, most elderly patients received antivirals and more than half of patients used antibiotics. The most common antiviral therapy was lopinavir and ritonavir per os plus recombinant human interferon- $a 2 b$ through aerosol inhalation. Several drug trials have shown that remdesivir [28] or favipiravir [29] may be effective in COVID-19, but due to limited samples and trial designs, further research is necessary. A recent study [30] showed that early antiviral therapy including lopinavir and ritonavir could improve the prognosis of COVID-19 patients. Before strong evidence for specific antivirals is available, lopinavir and ritonavir plus recombinant human interferon- $\alpha 2 b$ could still be used. As for elderly patients with increased neutrophils or procalcitonin, cough with yellow sputum, or patients in a severe condition, antibiotics were used to prevent or treat secondary infection, and the most common antibiotic was moxifloxacin in our patients. As for short-term outcomes, 3 deaths occurred in our cohort. On the date of analysis, 13 patients were still hospitalized and included 3 in a critically severe condition who were receiving ECOM or invasive ventilator support, meaning the mortality requires further follow-up.

This study has some limitations. First, more detailed elderly patient information is not available, such as the detailed medication history of comorbidities and the results of cytokines and coagulation factors. Therefore, it is hard to assess the drug influence on some laboratory abnormalities and explore the pathogenesis of SARS-CoV-2 in elderly patients. Second, due to the short time of follow-up, long-term outcomes of elderly patients are unknown, such as the influence on pulmonary function or possibility of lung fibrosis. Therefore, a prospective follow-up of these elderly patients is necessary.
In conclusion, elderly patients are liable to develop a severe or critically severe condition with SARS-CoV-2 infection. They could show atypical symptoms and multiple organ abnormalities. Old-old patients tend to have more complications than young-old patients during hospitalization, but with timely and effective treatment they could also achieve comparably good outcomes as young-old patients. Careful nursing, observation, and systemic treatment are very important in elderly patients.

\section{Statement of Ethics}

This study was approved by the Medical Ethical Committee of the Second Xiangya Hospital of Central South University (approval No. KL-2020020), which waived the requirement for patients' informed consent referring to the CIOMS guideline.

\section{Disclosure Statement}

The authors declare no conflicts of interest.

\section{Funding Sources}

This study was founded by the National Natural Science Foundation of China (No. 81370164 and No. 81670062), the National Natural Science Foundation of Hunan Province (No. 2015JJ4087), the National Key Clinical Specialist Construction Programs of China, and Innovative Major Emergent Project against the Outbreak of New Coronavirus Pneumonia in Hunan Province (No. 2020SK3014). The funder of the study had no role in the study design, data collection, data analysis, data interpretation, or writing of the report.

\section{Author Contributions}

Ting Guo, Qinxue Shen, Xiaoli Ouyang, Wei Guo, Wenlong $\mathrm{He}$, Jinhua Li, Yi Zhang, Yunnian Wang, and Hong Peng were responsible for data collection and analysis. Hong Luo, Zhiguo Zhou, Dingding Deng, Zhi Xiang, Minyan Jiang, Peng Huang, Wenlong Liu, and Minyong Liang were responsible for data collection and review of the manuscript. Ping Chen, Ting Guo, Qinxue Shen, Wei Guo, Wenlong He, Jinhua Li, Xudong Xiang, Zaimei Peng, and Hong Peng contributed substantially to the study design, interpretation, and writing of the report. 


\section{References}

1 Zhu N, Zhang D, Wang W, Li X, Yang B, Song J, et al.; China Novel Coronavirus Investigating and Research Team. A novel coronavirus from patients with pneumonia in China, 2019. N Engl J Med. 2020 Feb;382(8):727-33.

2 Huang C, Wang Y, Li X, Ren L, Zhao J, Hu Y, et al. Clinical features of patients infected with 2019 novel coronavirus in Wuhan, China. Lancet. 2020 Feb;395(10223):497-506.

3 WHO. Coronavirus disease (COVID-19) Pandemic. Available from: https://www.who.int/ emergencies/diseases/novel-coronavirus-2019.

4 WHO. General's opening remarks at the Mission briefing on COVID-19 - 12 March 2020. Available from: https:/www.who.int/dg/ speeches/detail/who-director-general-sopening-remarks-at-the-mission-briefingon-covid-19-12-march-2020.

5 Dawei W, Bo H, Chang H, et al. Clinical characteristics of 138 hospitalized patients with 2019 novel coronavirus-infected pneumonia in Wuhan, China. JAMA. 2020;323(11): 1061-69.

6 Yang X, Yu Y, Xu J, Shu H, Xia J, Liu H, et al. Clinical course and outcomes of critically ill patients with SARS-CoV-2 pneumonia in Wuhan, China: a single-centered, retrospective, observational study. Lancet Respir Med. 2020 May;8(5):475-81.

7 Zhou F, Yu T, Du R, Fan G, Liu Y, Liu Z, et al. Clinical course and risk factors for mortality of adult inpatients with COVID-19 in Wuhan, China: a retrospective cohort study. Lancet. 2020 Mar;395(10229):1054-62.

8 Tian S, Hu N, Lou J, Chen K, Kang X, Xiang $Z$, et al. Characteristics of COVID-19 infection in Beijing. J Infect. 2020 Apr;80(4):401-6.

9 WHO. Clinical management of severe acute respiratory infection when novel coronavirus $(\mathrm{nCoV})$ infection is suspected: interim guidance. Jan 28, 2020. Geneva: World Health Organization; 2020.

10 Chinese National Health Committee. The diagnostic and treatment guideline for novel coronavirus pneumonia (version seven). Available from: http://www.nhc.gov.cn/yzy$\mathrm{gj} / \mathrm{s} 7653 \mathrm{p} / 202003 / 46 \mathrm{c} 9294 \mathrm{a} 7 \mathrm{dfe} 4 \mathrm{cef} 80 \mathrm{dc} 7 \mathrm{f5}$ 912eb1989.shtml
11 Wu J, Liu J, Zhao X, Liu C, Wang W, Wang $\mathrm{D}$, et al. Clinical characteristics of imported cases of COVID-19 in Jiangsu Province: a multicenter descriptive study. Clin Infect Dis. 2020 Feb. DOI: 10.1093/cid/ciaa199.

12 Chen N, Zhou M, Dong X, Qu J, Gong F, Han $Y$, et al. Epidemiological and clinical characteristics of 99 cases of 2019 novel coronavirus pneumonia in Wuhan, China: a descriptive study. Lancet. 2020 Feb; 395(10223):507-13.

13 Song F, Shi N, Shan F, Zhang Z, Shen J, Lu H, et al. Emerging 2019 novel coronavirus (2019nCoV) pneumonia. Radiology. 2020 Apr; 295(1):210-7.

14 Zhang JJ, Dong X, Cao YY, Yuan YD, Yang YB, Yan YQ, et al. Clinical characteristics of 140 patients infected with SARS-CoV-2 in Wuhan, China. Allergy. 2020 Feb. DOI: 10.1111/all.14238.

15 Xu XW, Wu XX, Jiang XG, Xu KJ, Ying LJ, Ma $\mathrm{CL}$, et al. Clinical findings in a group of patients infected with the 2019 novel coronavirus (SARS-Cov-2) outside of Wuhan, China: retrospective case series. BMJ. 2020 Feb; 368:m606.

16 Norman DC, Yoshikawa TT. Fever in the elderly. Infect Dis Clin North Am. 1996 Mar; 10(1):93-9.

17 Zumla A, Chan JF, Azhar EI, Hui DS, Yuen KY. Coronaviruses - drug discovery and therapeutic options. Nat Rev Drug Discov. 2016 May;15(5):327-47.

18 Wong RS, Wu A, To KF, Lee N, Lam CW Wong CK, et al. Haematological manifestations in patients with severe acute respiratory syndrome: retrospective analysis. BMJ. 2003 Jun;326(7403):1358-62.

19 Qin L, Jing X, Qiu Z, Cao W, Jiao Y, Routy JP, et al. Aging of immune system: immune signature from peripheral blood lymphocyte subsets in 1068 healthy adults. Aging (Albany NY). 2016 May;8(5):848-59.
20 Suxin W, Qingjie Y, Shibing F, et al. Characteristics of lymphocyte subsets and cytokines in peripheral blood of 123 hospitalized patients with 2019 novel coronavirus pneumonia (NCP). medRxiv. 2020. DOI: https://doi. org/10.1101/2020.02.10.20021832.

21 Mikuła T, Sapuła M, Jabłońska J, Kozłowska J, Stańczak W, Krankowska D, et al. Significance of heparin-binding protein and D-dimers in the early diagnosis of spontaneous bacterial peritonitis. Mediators Inflamm. 2018 Sep;2018:1969108-14.

22 Gris JC, Bouvier S, Cochery-Nouvellon E, Faillie JL, Lissalde-Lavigne G, Lefrant JY. Fibrin-related markers in patients with septic shock: individual comparison of D-dimers and fibrin monomers impacts on prognosis. Thromb Haemost. 2011 Dec; 106(6):1228-30.

23 Jing L, Sumeng L, Jia L, et al. Longitudinal characteristics of lymphocyte responses and cytokine profiles in the peripheral blood of SARSCoV-2 infected patients. medRxiv. 2020. DOI: https://doi.org/10.1101/2020.02.16.20023671.

24 Slaats J, Ten Oever J, van de Veerdonk FL, Netea MG. IL-1 $\beta / \mathrm{IL}-6 / \mathrm{CRP}$ and IL-18/ferritin: distinct inflammatory programs in infections. PLoS Pathog. 2016 Dec;12(12):e1005973.

25 Franchini M. Hemostasis and aging. Crit Rev Oncol Hematol. 2006 Nov;60(2):144-51.

26 Mari D, Coppola R, Provenzano R. Hemostasis factors and aging. Exp Gerontol. 2008 Feb; 43(2):66-73.

27 Weinstein JR, Anderson S. The aging kidney: physiological changes. Adv Chronic Kidney Dis. 2010 Jul;17(4):302-7.

28 Grein J, Ohmagari N, Shin D, Diaz G, Asperges $\mathrm{E}$, Castagna A, et al. Compassionate use of remdesivir for patients with severe Covid-19. N Engl J Med. 2020 Apr. DOI: 10.1056/ NEJMoa2007016.

29 Chang C, Jianying H, Zhenshun C, et al. Favipiravir versus arbidol for COVID-19: a randomized clinical trial. medRxiv. 2020. DOI: https://doi.org/10.1101/2020.03.17.20037432.

30 Wu J, Li W, Shi X, Chen Z, Jiang B, Liu J, et al. Early antiviral treatment contributes to alleviate the severity and improve the prognosis of patients with novel coronavirus disease (COVID-19). J Intern Med. 2020 Mar. DOI: 10.1111/joim.13063. 